\title{
A Helmholtz-Lie Type Characterization of Ellipsoids, II
}

\author{
P. M. Gruber and M. Ludwig
}

Abteilung für Analysis, Technische Universität Wien, Wiedner Hauptstraße 8-10/1 142, A-1040 Vienna, Austria

pmgruber/mludwig@pop.tuwien.ac.at

\begin{abstract}
A closed convex surface $S$ in $\mathbb{E}^{d}$ is an ellipsoid if and only if for any $x, y \in S$ there is an affinity mapping $x$ onto $y$ and a neighborhood of $x$ in $S$ onto a neighborhood of $y$ in $S$.
\end{abstract}

\section{Introduction and Statement of Result}

1.1. The present articles deal with the problem of characterizing ellipsoids among all closed convex surfaces in Euclidean $d$-space $\mathbb{E}^{d}$ by local transformation properties. Using topological tools the answer for odd $d$ was obtained in the first article. Here the answer for all $d$ is given. More precisely, the following result is proved where the closed convex surface is the boundary of a compact convex subset of $\mathbb{E}^{d}$ with nonempty interior.

Theorem. Let $S$ be a closed convex surface in $\mathbb{E}^{d}$ with the following property $\mathcal{A}$ : to any pair of points $x, y \in S$ there corresponds an affine transformation $A_{x y}$ of $\mathbb{E}^{d}$ which maps $x$ onto $y$ and a suitable neighborhood $N_{x y}$ of $x$ in $S$ onto a neighborhood of $y$ in $S$. Then $S$ is an ellipsoid.

For related results we refer to the introduction of the first article. To the convexity results cited there we include Mäurer [11]. Related results in the context of differential geometry and affine differential geometry are due to Szabó [15] and Liu and Wang [9].

1.2. The proof relies heavily on results of Leichtweiss on floating bodies and of Blaschke and Petty in affine differential geometry. In particular, a characterization of ellipsoids due to Petty is needed utilizing the notions of affine distance and Santaló point. Moreover, use is made of a characterization of spheres by the property that their Gauss curvature is constant, which goes back to Liebmann, and of a characterization of 
convex sets due to Tietze. In addition, we use tools from the first article and in the case $d=2$ the solution of a functional equation is needed.

\section{General Tools and Preliminaries}

For concepts not explained below and results for which no reference is given we refer to [14]. Let $|\cdot|$, conv, relint, and det stand for volume, convex hull, relative interior with respect to a given surface, and determinant of the linear part of an affinity, respectively. $\|\cdot\|$ and $S^{d-1}$ denote the Euclidean norm and the unit sphere in $\mathbb{E}^{d}$.

Let $S$ be a closed convex surface in $\mathbb{E}^{d}$.

2.1. It is well known that:

(1) A convex function on an interval in $\mathbb{R}$ is almost everywhere twice differentiable.

2.2. The supporting function $h(S, \cdot)$ of $S$ is defined on $S^{d-1}$. Assume now that $S$ is smooth, i.e., of class $\mathcal{C}^{1}$. Then for $x \in S$ we denote by $n(S, x)$ the exterior normal unit vector of $S$ at $x$. Given $\delta>0$, let $C(S, x, \delta)$ be the cap of $S$ with center $x$ and height $\delta$, that is the part of $S$ between the supporting hyperplane of $S$ at $x$ and its translate by the vector $-\delta n(S, x)$. The base $B(S, x, \delta)$ of $C(S, x, \delta)$ is the convex hull of the intersection of $S$ with the translated supporting hyperplane. If $\mu=|\operatorname{conv} C(S, x, \delta)|$, then $\mu$ is called the volume of $C(S, x, \delta)$ and instead of $B(S, x, \delta)$ and $C(S, x, \delta)$ we write $B(S, x, \mu)$ and $C(S, x, \mu)$, respectively. For $S$ of class $\mathcal{C}^{2}$ let $\kappa(S, u)$ be the Gauss curvature of $S$ at the point(s) of $S$ with exterior normal unit vector $u$. Thus $\kappa(S, \cdot)$ is defined on $S^{d-1}$.

2.3. Let $S$ be of class $\mathcal{C}^{1}$. For $v>0$ a closed convex surface $S_{[v]}$ in the interior of $S$ is the floating surface of $S$ corresponding to $v$ if any supporting hyperplane of $S_{[v]}$ cuts off from $S$ a cap of volume $v$. The following result is well known:

(2) Let $S_{[v]}$ be a floating surface of $S$. Then the base of each cap of $S$ of volume $v$ touches $S_{[v]}$ at a unique point and this point is the centroid of the base.

In order to state the next result we introduce the following notion where $\varepsilon>0$. The surface $S$ is $\varepsilon$-smooth if for any $x \in S$ there is a (solid Euclidean) ball of radius $\varepsilon$ contained in conv $S$ which touches $S$ at $x$. If for each $x \in S$ there is a ball of radius $1 / \varepsilon$ containing $S$ and such that its boundary touches $S$ at $x$, then $S$ is called $\varepsilon$-strictly convex.

From Leichtweiss [6] we take the following proposition:

(3) Let $S$ be $\varepsilon$-smooth for a given $\varepsilon>0$ and strictly convex. Then there is a $\lambda>0$ such that for $0<v<\lambda$ the floating surface $S_{[v]}$ of $S$ exists, is of class $\mathcal{C}^{2}$, and the inequality $\kappa\left(S_{[\nu]}, \cdot\right)>0$ holds.

2.4. Elementary calculations yield the next result, compare [7].

(4) Let $S$ be of class $\mathcal{C}^{2}$ and let $L$ be a volume-preserving linear transformation of 
$\mathbb{E}^{d}$. Then, for each $x \in S$ and $u=n(S, x)$,

$$
n(L(S), L(x))=\frac{L^{-t}(u)}{\left\|L^{-t}(u)\right\|}=v, \text { say, }
$$

and

$$
\kappa(L(S), v)=\frac{\kappa(S, u)}{\left\|L^{-1}(u)\right\|^{d+1}} .
$$

Here $L^{-t}$ is the inverse of the adjoint transformation of $L$.

2.5. A closed set $U$ in $\mathbb{E}^{d}$ is said to have the local supporting property if, for each of its boundary points $x$, there is a hyperplane through $x$, such that all points of $U$ in a suitable neighborhood of $x$ are on the same side of the hyperplane, possibly on the hyperplane. A result of Tietze [16] implies the following:

(5) Let $U$ be a closed compact surface in $\mathbb{E}^{d}$ which is star-shaped with respect to the origin $o$, that is, each open ray starting at $o$ meets $U$ in precisely one point. If $U$ has the local supporting property, then it is a closed convex surface.

2.6. Let $S$ be of class $\mathcal{C}^{2}$ with $\kappa(S, \cdot)>0$ and assume that $o$ is in its interior. Generalizing a result of Blaschke [3], Petty [12] proved, for the affine distance,

(6) $a(S, u)=h(S, u) \kappa(S, u)^{-1 /(d+1)}$

from $o$ to the (unique) point $x \in S$ where $u=n(S, x)$, the formula

(7)

$$
a(S, u)^{d+1}=\lim _{\delta \rightarrow+0} \frac{d^{d+1}|\operatorname{conv}(\{o\} \cup B(S, x, \delta))|}{(d+1)^{d-1} \kappa_{d-1}^{2}|\operatorname{conv} C(S, x, \delta)|} .
$$

Here $\kappa_{d-1}$ is the volume of the unit ball in $\mathbb{E}^{d-1}$.

The Santalo point of $S$ is the point

(8) $\operatorname{san} S=\int_{S^{d-1}} u h(S, u)^{-(d+1)} d \sigma(u)$,

where $\sigma$ is the ordinary surface area measure on $S^{d-1}$ and the integral is to be understood componentwise.

The following characterization of ellipsoids is due to Petty [13]:

(9) Let $S$ be of class $\mathcal{C}^{2}$ with $\kappa(S, \cdot)>0$ where $o=\operatorname{san} S$. If $a(S, \cdot)$ is constant on $S^{d-1}$, then $S$ is an ellipsoid with center $o$.

2.7. It is well known that:

(10) If $S$ is of class $\mathcal{C}^{2}$ with $\kappa(S, \cdot)>0$, then

$$
\int_{S^{d-1}} \frac{u}{\kappa(S, u)} d \sigma(u)=o
$$


Also well known is the next result, of which a first version was given by Liebmann [8].

(11) Let $S$ be of class $\mathcal{C}^{2}$. If $\kappa(S, \cdot)$ is constant, then $S$ is a sphere.

\section{Proof of the Theorem}

Let $S$ be as stated in the theorem. The proof that $S$ is an ellipsoid is split into three parts: in Section 3.1 some necessary tools from the first article are cited and a lemma on $\varepsilon$ smoothness is proved. In Sections 3.2 and 3.3 we distinguish the cases where respectively there are and are not points $p, q \in S$ such that for corresponding affinities $A_{p q}$ and $B_{p q}$ the nonequality $\left|\operatorname{det} A_{p q}\right| \neq\left|\operatorname{det} B_{p q}\right|$ holds. In the former case we treat the cases $d=2$ and $d \geq 3$ separately.

\subsection{Tools from the First Article and a Lemma.}

3.1.1. From Sections $3.2,3.3,3.4 .2$, and 3.4 .3 of [5], respectively, we take the following propositions:

(12) $S$ is of class $\mathcal{C}^{1}$ and is strictly convex.

(13) $\operatorname{det} A_{x y} \neq 0$ for all $x, y \in S$.

(14) Assume that for each pair $x, y \in S$ the value of $\left|\operatorname{det} A_{x y}\right|$ is the same for all affinities $A_{x y}$. Then, given $p \in S$, the function

$$
x \rightarrow\left|\operatorname{det} A_{p x}\right|: x \in S
$$

is continuous and thus bounded between positive constants by (13).

(15) Assume that for each pair $x, y \in S$ the value of $\left|\operatorname{det} A_{x y}\right|$ is the same for all affinities $A_{x y}$. Then, given $p \in S$, there is a $\mu>0$ such that

$$
\begin{aligned}
C(S, p, \mu) & \subset N_{p x}, \\
A_{p x}(C(S, p, \mu)) & =C\left(S, x,\left|\operatorname{det} A_{p x}\right| \mu\right)
\end{aligned}
$$

for each $x \in S$ and suitable $A_{p x}$.

(16) Let $d \geq 3$ and assume that there are points $p, q \in S$ and corresponding affinities $A_{p q}$ and $B_{p q}$ with $\left|\operatorname{det} A_{p q}\right| \neq\left|\operatorname{det} B_{p q}\right|$. Then $S$ is an ellipsoid.

3.1.2. This subsection contains the proof of the following lemma:

(17) There is an $\varepsilon>0$ such that $S$ is $\varepsilon$-smooth and $\varepsilon$-strictly convex.

Since the proofs for $\varepsilon$-smoothness and for $\varepsilon$-strict convexity are very similar, only the former will be given. The first step is to show the following:

(18) For each $x \in S$ there is a ball $B_{x}$, with $x \in B_{x} \subset$ conv $S$.

Obviously, there is a point $p \in S$ and a ball $B$ such that $p \in B \subset$ conv $S$. Now, for any $x \in S$, by replacing $B$ by a suitable smaller ball which then is also denoted by $B$, if necessary, we may assume that $B \subset \operatorname{conv} N_{p x}$ where $N_{p x}$ is a neighborhood 
corresponding to $p, x$. Then

$$
x=A_{p x}(p) \in A_{p x}(B) \subset A_{p x}\left(\operatorname{conv} N_{p x}\right)=\operatorname{conv} A_{p x}\left(N_{p x}\right) \subset \operatorname{conv} S .
$$

Now choose a ball $B_{x}$ contained in the ellipsoid $A_{p x}(B)$ with $x \in B_{x}$. The proof of (18) is complete.

Clearly, we may assume that for each $x \in S$ the ball $B_{x}$ has maximum radius, say $\varepsilon_{x}$. A simple compactness argument then yields

(19) $S_{n}=\left\{x \in S: \varepsilon_{x} \geq 1 / n\right\}$ is closed in $S$ for $n=1,2, \ldots$

By (18),

(20) $S=\bigcup_{n=1}^{\infty} S_{n}$.

Since $S$ is (with the induced metric) a complete metric space, a version of the Baire category theorem together with (19) and (20) implies that

$$
\text { relint } S_{n} \neq \emptyset \text { for a suitable index } n \text {. }
$$

Let $p \in$ relint $S_{n}$. Obviously,

$$
A_{p x}\left(N_{p x} \cap \text { relint } S_{n}\right), x \in S, \text { is an open covering of } S .
$$

By the compactness of $S$ there are open neighborhoods $N_{1}, \ldots, N_{k}$ of $p$ in $S_{n}$ and nonsingular affinities $A_{1}, \ldots, A_{k}$ such that

$$
A_{1}\left(N_{1}\right), \ldots, A_{k}\left(N_{k}\right) \text { is an open covering of } S \text {. }
$$

Again, the compactness of $S$ in conjunction with Lebesgue's covering lemma then shows that there are sets

(21) $M_{1} \subset N_{1}, \ldots, M_{k} \subset N_{k}$, compact,

while still

(22) $A_{1}\left(M_{1}\right), \ldots, A_{k}\left(M_{k}\right)$ is a covering of $S$.

Since $M_{i} \subset N_{i} \subset S_{n}$, for $i=1, \ldots, k$, the definition of $S_{n}$ in (19) implies:

(23) For each $q \in M_{i}, i=1, \ldots, k$, there is a ball of radius $\varepsilon=1 / n$ in conv $S$ which touches $S$ at $q$.

Taking into account (12) and (21), by decreasing $\varepsilon$ if necessary, we may replace (23) by the following proposition:

(24) There is an $\varepsilon>0$ such that for each $q \in M_{i}, i=1, \ldots, k$, there is a ball $B_{q}$ of radius $\varepsilon$ with $q \in B_{q} \subset$ conv $N_{i}$.

Now (22), (24), and an argument which is slightly more complicated than the one that led to (18) give (17). (Here we have to deal with $k$ ellipsoids.) 
3.2. Case 1. We assume here that:

(25) There are points $p, q \in S$ and corresponding affinities $A_{p q}$ and $B_{p q}$ with $\left|\operatorname{det} A_{p q}\right| \neq\left|\operatorname{det} B_{p q}\right|$.

Our aim is to show that then

(26) $S$ is an ellipsoid.

Remark. Since the affinities which map neighborhoods of points on an ellipsoid onto neighborhoods on the ellipsoid are volume-preserving, this shows that Case 1 actually cannot hold.

3.2.1 $(d=2)$. At first it is shown that a convex arc which properly contains an affine image of itself must contain an arc of a conic. The proof of this lemma in essence consists of the solution of a functional equation. The lemma together with assumption (25) then easily leads to (26).

We first show the following lemma:

(27) Let $p \in S$ and let $A$ be an arc of $S$ starting at $p$. Assume that $C_{p p} \neq i d$ is an affinity with det $C_{p p}>0$ such that $C_{p p}(A)$ is also an $\operatorname{arc}$ in $S$ which starts at $p$ in the same direction as $A$. Then a subarc of $A$ is an arc of a conic.

At first $A$ and $C_{p p}(A)$ are represented in a suitable Cartesian coordinate system: choose $p$ as the origin, let the supporting line of $S$ at $p$ (which is unique by (12)) be the first coordinate axis such that $A$ starts in the direction of the positive axis and let the positive second axis point into the half-plane containing $S$. The coordinates in this system are denoted $s, t$.

Clearly, we may represent a subarc of $A$ which starts at $p$ in the form

$$
t=f(s) \quad \text { or } \quad s=g(t)
$$

with suitable functions $f, g$. It follows from (1) and the property $\mathcal{A}$ of $S$ that $S$ is everywhere twice differentiable. Hence

(28) $f(s) \sim \alpha s^{2}$ as $s \rightarrow+0$ and thus $g(t) \sim \beta t^{1 / 2}$ as $t \rightarrow+0$,

where $\alpha>0$ by (17) and $\beta=\alpha^{-1 / 2}$.

Since the arcs $A$ and $C_{p p}(A)$ on $S$ both start at (the origin) $p$ in the direction of the positive first coordinate axis, the affinity $C_{p p}$ maps the positive axis onto itself. Hence we may represent $C_{p p}$ in the form

$$
\left(\begin{array}{l}
s \\
t
\end{array}\right) \rightarrow\left(\begin{array}{r}
a s+b t \\
c t
\end{array}\right) \quad \text { for } \quad\left(\begin{array}{l}
s \\
t
\end{array}\right) \in \mathbb{R}^{2}
$$

with suitable $a>0, b, c$. Clearly, $\operatorname{det} C_{p p}=a c>0$ implies that $c>0$. By replacing $C_{p p}$ by $C_{p p}^{-1}$ and writing $C_{p p}$ for $C_{p p}^{-1}$, if necessary, we may assume that

(29) $0<a \leq 1, c>0$. 
$C_{p p}$ maps $(g(t), t)^{t}$ onto $(a g(t)+b t, c t)^{t}$. For small $t>0$ the latter point is also on the subarc of $A$ which is represented by $g(\cdot)$. Thus

(30) $a g(t)+b t=g(c t)$ for small $t>0$,

and therefore

$$
a \beta t^{1 / 2}+b t \sim c^{1 / 2} \beta t^{1 / 2} \quad \text { as } \quad t \rightarrow+0
$$

by (28). Hence $a=c^{1 / 2}$ or $c=a^{2}$. Thus, if $a=1$ we have $c=1$ and (30) yields $b=0$, that is, $C_{p p}=i d$, a contradiction. Hence $a \neq 1$ and instead of (29) the sharper statement

(31) $0<a<1, c=a^{2}$

holds.

The final step in the proof of (27) is to show that

(32) $g(t)=\beta t^{1 / 2}-(b / a(1-a)) t$ for small $t>0$.

(30), (31), and (28) show that, for small $t>0$,

$$
\begin{aligned}
& g(t)=\frac{1}{a} g\left(a^{2} t\right)-\frac{b}{a} t \\
& =\frac{1}{a}\left\{\frac{1}{a} g\left(a^{4} t\right)-\frac{b}{a} a^{2} t\right\}-\frac{b}{a} t=\frac{1}{a^{2}} g\left(a^{4} t\right)-\frac{b}{a}(1+a) t \\
& =\frac{1}{a^{n}} g\left(a^{2 n} t\right)-\frac{b}{a}\left(1+a+\cdots+a^{n-1}\right) t=\frac{1}{a^{n}} g\left(a^{2 n} t\right)-\frac{b}{a} \frac{1-a^{n}}{1-a} t \\
& \rightarrow \beta t^{1 / 2}-\frac{b}{a(1-a)} t \quad \text { as } n \rightarrow \infty \text {, }
\end{aligned}
$$

which concludes the proof of (32).

Since the arc of $S$ defined by $g(\cdot)$ where $t>0$ is small is a conic arc by (32), the proof of (27) is complete.

Having proved (27), the proof of (26) is simple. Note assumption (25) and let $C_{p p}=$ $A_{p q}^{-1} B_{p q}$. By replacing $C_{p p}$ by $C_{p p}^{2}$ and writing $C_{p p}$ for $C_{p p}^{2}$, if necessary, we may suppose that

(33) $0<\operatorname{det} C_{p p} \neq 1$ and thus, in particular, $C_{p p} \neq i d$.

Clearly, $C_{p p}$ maps $p$ onto $p$ and, for a suitable $\operatorname{arc} A$ in $S$ starting at $p, C_{p p}(A)$ is also an arc of $S$ starting at $p$. The strict convexity of $S$ (see (12)) and $\operatorname{det} C_{p p}>0$ (see (33)) imply that $A$ and $C_{p p}(A)$ both start in the same direction. Thus an application of (27) shows that $S$ contains an arc of a conic. The transformation property $\mathcal{A}$, the compactness of $S$, and the fact that two overlapping arcs of conics are actually on the same conic then implies that $S$ is a conic itself. Being bounded, it is an ellipse, concluding the proof of (26).

3.2.2 ( $d \geq 3$ ). Assumption (25) together with (16) immediately yield (26). 
3.3. Case 2. We now assume that:

(34) For each pair $x, y \in S$ the value of $\left|\operatorname{det} A_{x y}\right|$ is the same for all corresponding affinities $A_{x y}$.

Again, our aim is to prove that

(35) $S$ is an ellipsoid.

As a first step it is shown that $\left|\operatorname{det} A_{x y}\right|=1$ for all $x, y \in S$. Then we prove that the floating surfaces $T=S_{[v]}$ also have property $\mathcal{A}$. Next, to each $T$ we assign a closed star-shaped surface $U$. (It is perhaps worth noting that $T$ is a dilatation of the curvature image of $U$, compare [10].) It then turns out that $U$ also has property $\mathcal{A}$ but with linear affinities. This yields in particular, using Tietze's theorem (5), that $U$ is a closed convex surface. Hence the floating surfaces $V=U_{[\nu]}$ of $U$ may be considered. They, again, have property $\mathcal{A}$ where the affinities are linear. A further property of the $V$ 's deals with the affine distance. This permits the application of Petty's characterization (9) of ellipsoids. Hence each $V$ is an ellipsoid. Then, going back from $V$ to $U$, from $U$ to $T$, and then to $S$, we see that $S$ is also an ellipsoid.

3.3.1. This subsection contains the proof that

(36) $\left|\operatorname{det} A_{p x}\right|=1$ for all $p, x \in S$.

Property $\mathcal{A}$ and assumption (34) yield the following proposition:

(37) Let $p, z, y \in S$ and let $A_{p z}, A_{z y}, A_{p y}$ be corresponding affinities. Then $\left|\operatorname{det} A_{p y}\right|$ $=\left|\operatorname{det} A_{p z}\right|\left|\operatorname{det} A_{z y}\right|$.

Let $p \in S$. Assumption (34) together with (14) implies that there is a $q \in S$ such that

$$
\left|\operatorname{det} A_{p q}\right|=\max \left\{\left|\operatorname{det} A_{p z}\right|: z \in S\right\} \text {. }
$$

Thus

(38) $\left|\operatorname{det} A_{p z}\right| \leq\left|\operatorname{det} A_{p q}\right|$ for all $z \in S$.

Let $x \in S$ and consider $A_{q x}$. The affinity $A_{q x}$ maps a neighborhood $N_{q x}$ of $q$ in $S$ onto a neighborhood $N_{x}$ of $x$ in $S$. For any $y \in N_{x}$ there is a point $z \in N_{q x}$ with $A_{q x}(z)=y$. By (34) we thus have

$$
\left|\operatorname{det} A_{z y}\right|=\left|\operatorname{det} A_{q x}\right| \text {. }
$$

This, (37), and (38) together then show

$$
\left|\operatorname{det} A_{p y}\right|=\left|\operatorname{det} A_{p z}\right|\left|\operatorname{det} A_{z y}\right| \leq\left|\operatorname{det} A_{p q}\right|\left|\operatorname{det} A_{q x}\right|=\left|\operatorname{det} A_{p x}\right| \quad \text { for all } \quad y \in N_{x} .
$$

The function $x \rightarrow\left|\operatorname{det} A_{p x}\right|$ thus has a local maximum at any point $x \in S$. Since this function is continuous by (34) and (14), it is a constant. Taking $x=p$, it follows that this constant is 1 , concluding the proof of (36). 
3.3.2. By (3) and (17),

(39) there is a $\lambda>0$ such that $T=S_{[\nu]}$ exists, is of class $\mathcal{C}^{2}$, and $\kappa\left(S_{[\nu]}, \cdot\right)>0$ for $0<v<\lambda$.

Since $\left|\operatorname{det} A_{x y}\right|=1$ for all $x, y \in S$ by (36), the following is a simple consequence of (15), where for $A_{x y}$ we take $A_{p y} A_{p x}^{-1}$ :

(40) There is a $\mu>0$ such that

$$
A_{x y}(C(S, x, \mu))=C(S, y, \mu)
$$

for all $x, y \in S$ and suitable $A_{x y}$.

In the following when we write $A_{x y}$ it is to be understood that $A_{x y}$ is a volume-preserving affinity which satisfies (40).

Our aim in this subsection is to show that

(41) $T=S_{[\nu]}$ has property $\mathcal{A}$ for $0<\nu<\min \{\lambda, \mu\}$ where the affinities are volume-preserving.

For the proof of (41) it is sufficient to verify the following:

(42) Let $0<v<\min \{\lambda, \mu\}$ and $u, v \in T=S_{[v]}$ be chosen. Since by (12) and (39) the surfaces $S$ and $T$ are smooth and strictly convex, there are unique $x, y \in S$ with $n(S, x)=n(T, u)$ and $n(S, y)=n(T, v)$. Then there is a neighborhood $M$ of $u$ in $T$ and a volume-preserving affinity $B_{u v}\left(=A_{x y}\right)$ which maps $u$ onto $v$ and $M$ into $T$.

The supporting hyperplane of $T$ at $u$ cuts off from $S$ the cap $C(S, x, v)$ of volume $v$. Since $v<\mu$, this cap is contained in relint $C(S, x, \mu)$. Thus, since $T$ is smooth and strictly convex (see (39)), there is a neighborhood $M$ of $u$ in $T$ such that:

(43) For each $w \in M$ the following hold: if $z \in S$ is chosen (uniquely) such that $n(S, z)=n(T, w)$, then

(a) the supporting hyperplane of $T$ at $w$ cuts off from $S$ the cap $C(S, z, v)$ of volume $v$, where

(b) $C(S, z, v) \subset C(S, x, \mu)$, and

(c) $w$ is the centroid of the base $B(S, z, v)$.

(a) and (b) are clear and (c) is implied by (2).

The proof that $B_{u v}=A_{x y}$ maps $u$ onto $v$ in essence is a special case of the proof that $B_{u v}$ maps $M$ into $T$. Hence only the latter will be given: let $w \in M$ and choose $z \in S$ such that $n(S, z)=n(T, w)$. By (43), $w$ is the centroid of the base $B(S, z, v)$ of the cap $C(S, z, v)$. Since $A_{x y}$ is nonsingular, $A_{x y}(w)$ is the centroid of the base of the cap

$$
A_{x y}(C(S, z, v))=C\left(S, A_{x y}(z), v\right) \subset S,
$$

see the definition of $M,(43)$, and (40). This implies that $B_{u v}(w)=A_{x y}(w)$ is the point where the base of the cap $C\left(S, A_{x y}(z), v\right)$ touches $T$. Thus $B_{u v}(w) \in T$, concluding the proof of (42) and thus of (41). 
3.3.3. The next aim is to establish the following proposition:

(44) Let $T\left(=S_{[v]}\right)$ be a closed convex surface of class $\mathcal{C}^{2}$ with $\kappa(T, \cdot)>0$ which has property $\mathcal{A}$. Define a closed surface $U$, star-shaped with respect to $o$, by

$$
U=\left\{\kappa(T, t)^{-1 /(d+1)} t: t \in S^{d-1}\right\} .
$$

Then $U$ has property $\mathcal{A}$ where the affinities are volume-preserving and linear and $U$ is convex with $o$ in its interior.

For the proof that $U$ has property $\mathcal{A}$ it is sufficient to prove the following:

(45) Let $r, s \in S^{d-1}$ and choose (unique) $u, v \in T$ such that $r=n(T, u)$ and $s=n(T, v)$. Then there is a neighborhood $N$ of $\kappa(T, r)^{-1 /(d+1)} r$ in $U$ and a volume-preserving linear transformation $L_{r s}^{-t}\left(L_{r s}\right.$ is the linear part of $\left.B_{u v}\right)$ which maps $\kappa(T, r)^{-1(d+1)} r$ onto $\kappa(T, r)^{-1 /(d+1)} s$ and $N$ into $U$.

By the assumptions in (44) there is a neighborhood $M$ of $u$ in $T$ and a volumepreserving affinity $B_{u v}$ mapping $u$ onto $v$ and $M$ into $T$. The assumptions in (44) also show that $T$ is smooth and strictly convex. Hence the exterior normal unit vectors of $T$ at the points of $M$ form a neighborhood of $r=n(T, u)$ in $S^{d-1}$. As $t$ ranges over this neighborhood, the vectors $\kappa(T, t)^{-1 /(d+1)} t$ form a neighborhood $N$ of $\kappa(T, r)^{-1 /(d+1)} r$ in $U$. In order to show that $L_{r s}^{-t}$ maps $N$ into $U$, let $\kappa(T, t)^{-1 /(d+1)} t \in N$. By the definitions of $N$ and $U$ there is a $w \in M$ with $t=n(T, w)$. Now apply (4):

$$
\begin{aligned}
L_{r s}^{-t}\left(\kappa(T, t)^{-1 /(d+1)} t\right) & =\kappa(T, t)^{-1 /(d+1)} L_{r s}^{-t}(t) \\
& =\left(\frac{\kappa(T, t)}{\left\|L_{r s}^{-t}(t)\right\|^{d+1}}\right)^{-1 /(d+1)} \frac{L_{r s}^{-t}(t)}{\left\|L_{r s}^{-t}(t)\right\|} \\
& =\kappa\left(L_{r s}(T), \frac{L_{r s}^{-t}(t)}{\left\|L_{r s}^{-t}(t)\right\|}\right)^{-1 /(d+1)} n\left(L_{r s}(T), L_{r s}(w)\right) \\
& =\kappa\left(B_{u v}(T), n\left(B_{u v}(T), B_{u v}(w)\right)\right)^{-1 /(d+1)} n\left(B_{u v}(T), B_{u v}(w)\right) \\
& =\kappa\left(T, n\left(T, B_{u v}(w)\right)\right)^{-1 /(d+1)} n\left(T, B_{u v}(w)\right) \in U,
\end{aligned}
$$

since $B_{u v}(w) \in T$. Thus $L_{r s}^{-t}(N) \subset U$. For $w=u$ we have $t=n(T, w=u)=r$ and for $v=B_{u v}(u)$ we have $n(T, v)=s$. Hence

$$
L_{r s}^{-t}\left(\kappa(T, r)^{-1 /(d+1)} r\right)=\kappa(T, s)^{-1 /(d+1)} s .
$$

Since $L_{r s}^{-t}$ is volume-preserving, the proof of (45) is finished.

Now we show that

(46) $U$ is convex with $o$ in its interior.

Choose $p \in U$ having maximum distance from $o$. The hyperplane through $p$ orthogonal to the vector $p$ supports $U$ locally at $p$ (even globally, but we do not need this). The transformation property $\mathcal{A}$ of $U$ (see (45)) then implies that $U$ is supported locally at each of its points. Thus (5) shows that $U$ is convex. By definition of $U$ (see (44)), $o$ is in the interior of $U$, concluding the proof of (46).

Having proved (45) and (46), proposition (44) follows. 
Taking into account (44), the argument of Section 3.3.2 shows that:

(47) For each sufficiently small $v>0$ the floating surface $V=U_{[v]}$ exists, is of class $\mathcal{C}^{2}$ with $\kappa(V, \cdot)>0$, has property $\mathcal{A}$ where the affinities are (volume-preserving and) linear, and $o$ is in the interior of $V$.

3.3.4. This subsection is devoted to the proof of the following proposition:

(48) Let $V\left(=U_{[v]}\right)$ be a closed convex surface of class $\mathcal{C}^{2}$ with $\kappa(V, \cdot)>0$ and $o$ in its interior which has property $\mathcal{A}$ where the affinities are (volume-preserving and) linear. Then $V$ is an ellipsoid with center $o$.

In the proof of (48) we first show that the affine distance from $o$,

(49) $a(V, \cdot)$ is constant on $V$.

Choose $x, y \in V$ and let $u=n(V, x), v=n(V, y)$. For all sufficiently small $\delta>0$ the cap $C(V, x, \delta)$ is mapped by a suitable volume-preserving linear transformation $L_{x y}$ onto a cap of the form $C(V, y, \varepsilon)$ of the same volume. The assumptions in (48) imply that $V$ is smooth and strictly convex. Hence $\varepsilon=\varepsilon(\delta) \rightarrow 0$ as $\delta \rightarrow+0$. Clearly, $L_{x y}$ maps the cone conv $(\{o\} \cup B(V, x, \delta))$ onto the cone conv $(\{o\} \cup B(V, y, \varepsilon))$. Therefore both cones have the same volume. This, holding for all sufficiently small $\delta>0$, together with (7) yield $a(V, u)=a(V, v)$, concluding the proof of (49).

From (8), (6), (49), and (10) it follows, for the Santaló point of $V$, that

$$
\begin{aligned}
\operatorname{san} V & =\int_{S^{d-1}} u h(V, u)^{-(d+1)} d \sigma(u)=\int_{S^{d-1}} u a(V, u)^{-(d+1)} \kappa(V, u)^{-1} d \sigma(u) \\
& =\text { const } \int_{S^{d-1}} \frac{u}{\kappa(V, u)} d \sigma(u)=o .
\end{aligned}
$$

Combining this with (49), we see that the affine distance from the Santaló point $o$ of $V$ is constant on $V$. Note that $V$ is of class $\mathcal{C}^{2}$, see (47). Hence an application of Petty's characterization (9) shows that $V$ is an ellipsoid with center $o$. This concludes the proof of (48).

3.3.5. Now we make use of what has been proved in earlier subsections to show (35), i.e., $S$ is an ellipsoid.

For sufficiently small $v>0$ the floating surfaces $V=U_{[v]}$ exist and are ellipsoids with center $o$, see (47) and (48). Clearly, they approximate $U$ arbitrarily closely from the interior as $v \rightarrow+0$. Hence

(50) $U$ is an ellipsoid with center $o$.

Next it is shown that

(51) $T\left(=S_{[\nu]}\right)$ is an ellipsoid for all sufficiently small $v>0$.

The floating surface $T=S_{[v]}$ exists for all sufficiently small $v>0$, compare (39). For such $v$ the corresponding closed convex surface $U$ is also an ellipsoid and its center is $o$ by (44) and (50). Let $L^{-t}$ be a volume-preserving linear transformation which transforms 
$U$ into a sphere with center $o$. Using (4) and (44) the following relation obtains:

$$
\begin{aligned}
L^{-t}(U) & =\left\{L^{-t}\left(\kappa(T, t)^{-1 /(d+1)} t\right): t \in S^{d-1}\right\} \\
& =\left\{\kappa(T, t)^{-1 /(d+1)} L^{-t}(t): t \in S^{d-1}\right\} \\
& =\left\{\left(\frac{\kappa(T, t)}{\left\|L^{-t}(t)\right\|^{d+1}}\right)^{-1 /(d+1)} \frac{L^{-t}(t)}{\left\|L^{-t}(t)\right\|}: t \in S^{d-1}\right\} \\
& =\left\{\kappa(L(T), s)^{-1 /(d+1)} s: s \in S^{d-1}\right\},
\end{aligned}
$$

see [10]. Since $L^{-t}(U)$ is a sphere with center $o$, this can hold only if $\kappa(L(T), \cdot)$ is constant on $S^{d-1}$. Now, noting (39), proposition (11) implies that $L(T)$ is a sphere. This concludes the proof of (53).

The floating surfaces $T=S_{[v]}$ approximate $S$ arbitrarily closely as $v \rightarrow+0$. This together with (51) shows that $S$ is an ellipsoid. Thus the proof of (35) is complete.

3.4. Having shown that $S$ is an ellipsoid, the proof of the theorem is complete.

\section{Acknowledgments}

In a first version of this article we proved the theorem for $d=2$ and for $d \geq 3$ under the additional assumption that $\left|\operatorname{det} A_{x y}\right|=1$ for all $x, y \in S$. Then Dr. Daniel Hug pointed out that this assumption may easily be eliminated; see Section 3.3.1 for an argument similar to his. We are obliged to Dr. Hug for these most helpful comments. Many thanks are also due to Professor John Chalk for his numerous hints.

\section{References}

1. Auerbach, H.: Sur une propriété caracteristique de l'ellipsoide, Studia Math. 9 (1940), 17-22.

2. Blaschke, W.: Über affine Geometrie XII: von den Eiflächen, Leipziger Ber. 70 (1918), 18-37; Gesammelte Werke, vol. 4, Thales-Verlag, Essen, 1985, pp. 157-176.

3. Blaschke, W.: Vorlesungen über Differentialgeometrie II, Springer-Verlag, Berlin, 1923.

4. Busemann, H.: Timelike spaces, Dissertationes Math. (Rozprawy Mat.) 53 (1967), 52.

5. Gruber, P. M.: A Helmholtz-Lie type characterization of ellipsoids, I, Discrete Comput. Geom. 13 (1995), $517-527$

6. Leichtweiss, K.: Zur Affinoberfläche konvexer Körper, Manuscripta Math. 56 (1986), 429-464.

7. Leichtweiss, K.: Über einige Eigenschaften der Affinoberfläche beliebiger konvexer Körper, Resultate Math. 13 (1988), 255-282.

8. Liebmann, H.: Über die Verbiegung der geschlossenen Flächen positiver Krümmung, Math. Ann. 53(1900), 81-112.

9. Liu, H., Wang, C.: Centroffinely homogeneous surfaces in $R^{3}$, Beitr. Geom. Algebra 35 (1994), 109-117.

10. Lutwak, E.: Selected affine isoperimetric inequalities, in: Handbook of Convex Geometry, vol. A, NorthHolland, Amsterdam, 1993, pp. 151-176.

11. Mäurer, H.: Parabolische Kollineationen, die eine ebene konvexe Menge invariant lassen, Geom. Dedicata 58 (1995), 15-20.

12. Petty, C.: Geominimal surface area, Geom. Dedicata 3 (1974), 77-97.

13. Petty, C.: Affine isoperimetric problems, Ann. N.Y. Acad. Sci. 440 (1985), 113-127. 
14. Schneider, R.: Convex Bodies: the Brunn-Minkowski Theory, Cambridge University Press, Cambridge, 1993.

15. Szabó, Z. I.: Generalized spaces with many isometries, Geom. Dedicata 11 (1981), 369-383.

16. Tietze, H.: Bemerkungen über konvexe und nicht-konvexe Figuren, J. Reine Angew. Math. 160 (1929), $67-69$.

Received November 3, 1994, and in revised form May 23, 1995. 